Article

\title{
Competitive Centipede Games: Zero-End Payoffs and Payoff Inequality Deter Reciprocal Cooperation
}

\author{
Eva M. Krockow *, Briony D. Pulford and Andrew M. Colman \\ Department of Neuroscience, Psychology and Behaviour, University of Leicester, \\ Leicester LE1 7RH, UK; E-Mails: bdp5@le.ac.uk (B.D.P.); amc@le.ac.uk (A.M.C.) \\ * Author to whom correspondence should be addressed; E-Mail: emk12@le.ac.uk; \\ Tel.: +44-(0)116-229-7084; Fax: +44-(0)116-229-7196.
}

Academic Editor: Ulrich Berger

Received: 2 July 2015 / Accepted: 14 August 2015 / Published: 18 August 2015

\begin{abstract}
Reciprocal cooperation can be studied in the Centipede game, in which two players alternate in choosing between a cooperative GO move and a non-cooperative STOP move. GO sustains the interaction and increases the player pair's total payoff while incurring a small personal cost; STOP terminates the interaction with a favorable payoff to the defector. We investigated cooperation in four Centipede games differing in their payoffs at the game's end (positive versus zero) and payoff difference between players (moderate versus high difference). The games shared the same game-theoretic solution, therefore they should have elicited identical decision patterns, according to orthodox game theory. Nevertheless, both zero-end payoffs and high payoff inequality were found to reduce cooperation significantly. Contrary to previous predictions, combining these two factors in one game resulted in a slight weakening of their independent deterrent effects. These findings show that small changes in the payoff function have large and significant effects on cooperation, and that the effects do not combine synergistically.
\end{abstract}

Keywords: centipede game; backward induction; take-it-or-leave-it game; end-game effects; cooperation; reciprocity 


\section{Introduction}

Reciprocal interactions characterized by a repeated pattern of give-and-take underlie many human relationships. The following example should not be taken too literally but rather be considered as an archetype of a wide range social relationships: Two neighbors with adjacent properties take turns, once a week, tending to an apple tree growing on the border which separates their gardens. With each week of cultivation, the number of apples on the tree increases, and existing apples ripen, thus improving the prospective value of the harvest. This process continues as long as both neighbors continue to cooperate, and it terminates as soon as one of them defects by harvesting the crop. In this case, the defector secures a larger share of fruit, leaving a comparatively small share to the other person. If neither neighbor chooses to defect, then the process comes to a natural end once the fruit have reached full ripeness. In this second case, the individual whose turn it is at the time takes the larger share. The same basic strategic structure is present in a very wide variety of human interactions, including interpersonal relationships characterized by repeated, alternating opportunities for reciprocal acts of cooperation, economic and business relationships with recurrent opportunities for turn-taking assistance or favors, and political interactions with sequential opportunities for the exchange of support in voting assemblies, for example.

In the apple harvesting example, even small variations of the collaborative reciprocal relationship could turn it into a new, much more competitive interaction. One such variation could be the introduction of a deadline by which the crop had to be harvested in order to prevent the fruit from rotting and leaving both neighbors with nothing. Such a deadline would make defection-at some point during the interaction - virtually inevitable. Hence, in contrast to the decision whether to defect, in this scenario individuals would have to decide when to defect. Another variation could be to increase the inequality in crop distribution between the two neighbors, in the most extreme case allocating the total crop to the defector. By increasing differences in relative gains, the roles of "winner" and "loser" become more pronounced and every cooperative action becomes more risky.

The harvest decision context and its two competitive variations can be modeled with variations of Rosenthal's [1] Centipede game, a two-player game of complete and perfect information. Rosenthal's original version, which he did not name in his original article, was of the type shown in Figure 1. Players $\mathrm{A}$ and $\mathrm{B}$ alternate in choosing between a cooperative GO move and a non-cooperative (defecting) STOP move at their respective decision nodes. Whereas GO serves to sustain the relationship, STOP terminates the game and leads down to an exit node at the bottom of the game tree, where both players' final payoffs are displayed. The game was first named in print by Binmore [2] after its visual resemblance to a multi-legged insect. At a workshop in Haifa organized by the Israeli economist Joseph Greenberg in 1988, Robert Aumann presented a competitive version belonging to the type shown in Figure 2, in which both players are faced with zero payoffs at the end of the interaction if neither chooses to defect. We shall call games of this type Zero-end Centipede games. The competitive version shown in Figure 3, in which a player who defects takes the entire payoff pot that has accumulated up to that point, leaving nothing to the co-player, was introduced by Reny [3], who named games of this type Take-it-or-leave-it Centipede games (Huck and Jehiel [4] called them Take-or-pass games). Finally, a game version combining both competitive features-Zero-end and Take-it-or-leave it payoff function-is displayed in Figure 4. 


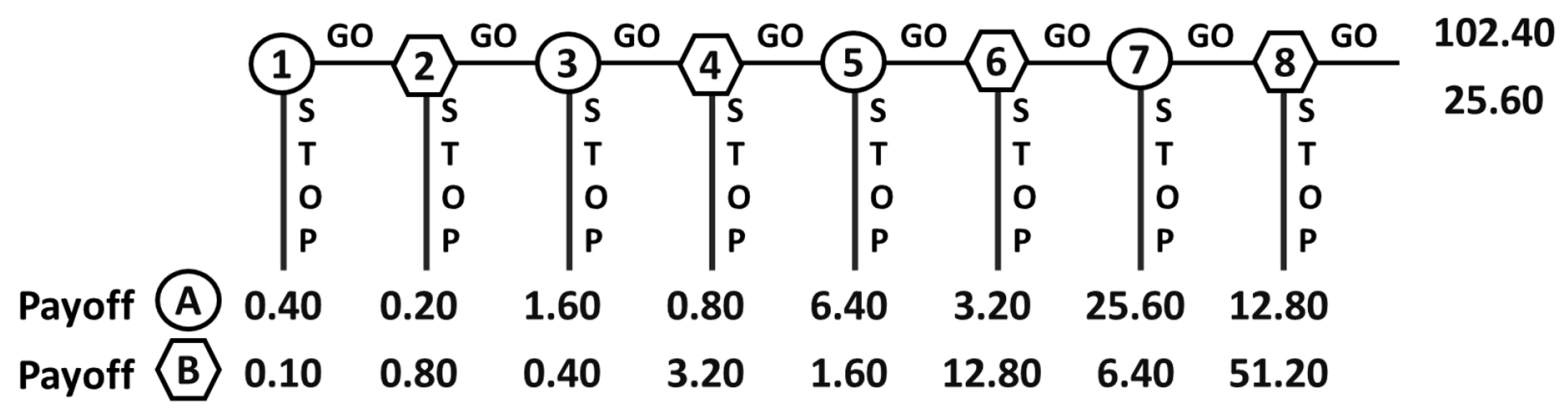

Figure 1. Exponential Centipede game with positive payoffs at terminal exit node.

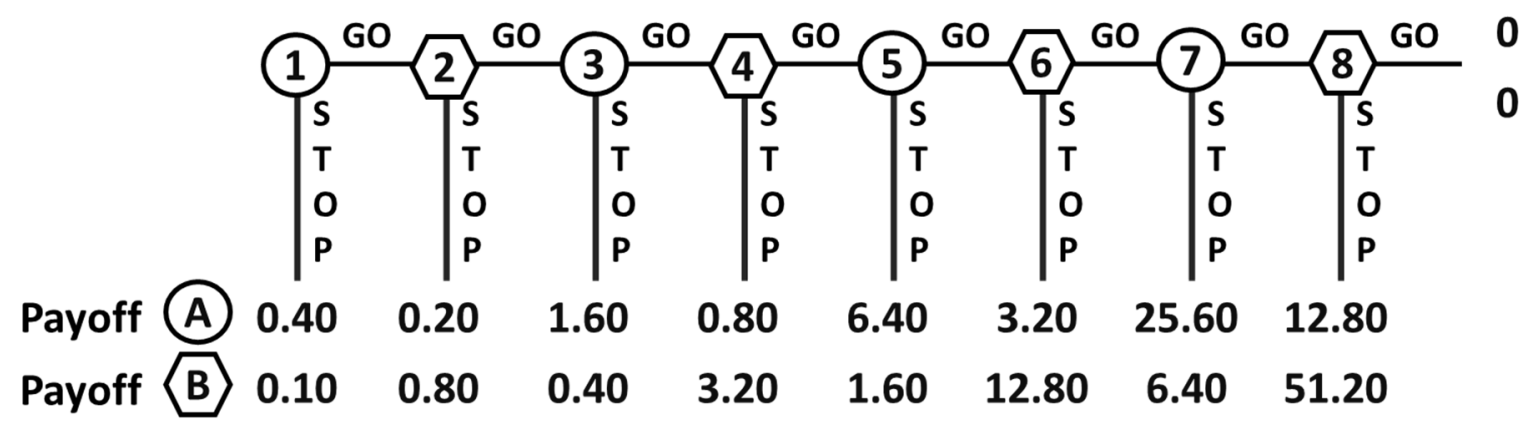

Figure 2. Exponential Zero-end Centipede game.

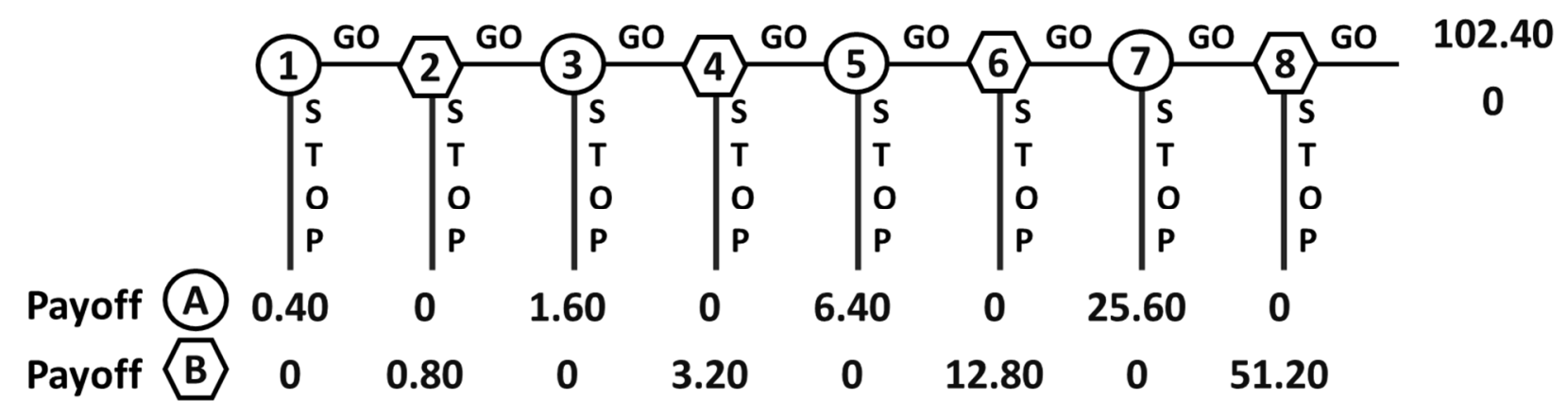

Figure 3. Take-it-or-leave-it Centipede game with positive payoffs at terminal exit node.

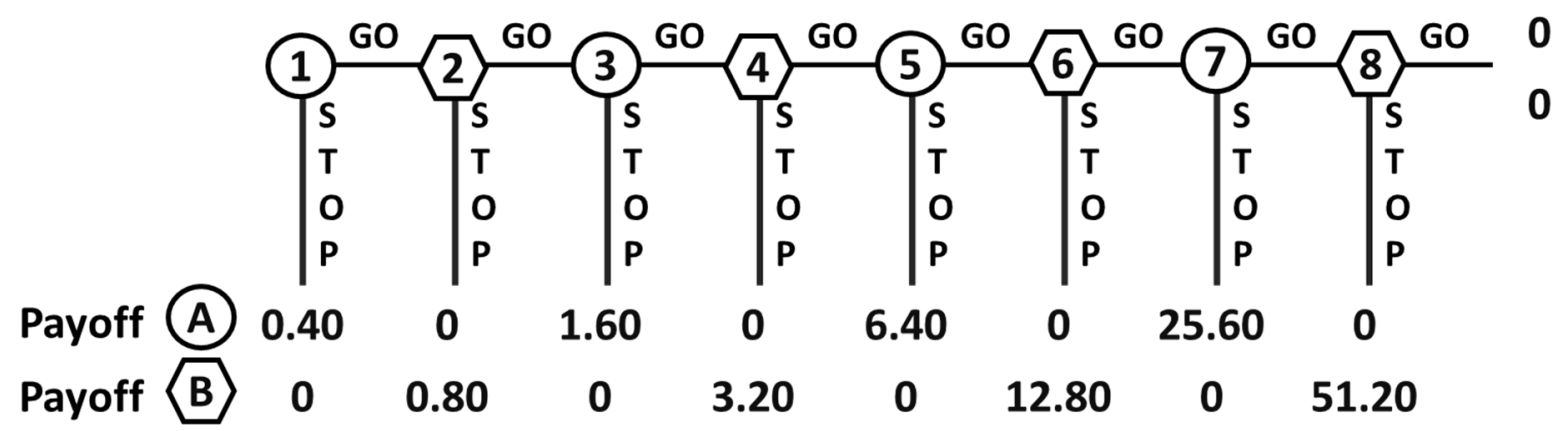

Figure 4. Take-it-or-leave-it plus Zero-end Centipede game.

The subgame perfect Nash equilibrium solution of the Centipede game - in all its variations - is an immediate STOP move by Player A at Node 1, and this is proved with a well-known backward induction 
(BI) argument. Referring for convenience just to the version shown in Figure 1, the BI reasoning begins at the game's final decision node, Node 8, where Player B faces a choice between GO, leading to the natural end and a personal payoff of 25.60, and STOP, earning 51.20. Player B, who is assumed to be a rational payoff maximizer, will therefore STOP at Node 8. Assuming that Player A is also a rational payoff maximizer and that the specification of the game and the rationality of both players are common knowledge, at Node 7 Player A will anticipate B's defection and will therefore choose GO move at Node 7, and the argument unfolds all the way back to the beginning of the game, where Player A will defect immediately.

The game-theoretic solution, despite appearing logically compelling and being supported by formal mathematical proofs [5-8], is highly counterintuitive. Comparing the outcomes of 0.40 and 102.40, the prospect of the higher amount should make a STOP at the first node seem unattractive. Even in the more competitive versions of the game (Figures 2 and 3), much higher payoffs can be obtained through mutual cooperation. Indeed, a growing body of empirical evidence has shown strong and reliable deviations from the equilibrium solution with little and inconsistent learning across games [9-12]. In McKelvey and Palfrey's [10] first empirical study of the game, only $0.7 \%$ of players in their six-node game stopped at the first decision node, and $1.4 \%$ continued until the natural end of the game, representing a strong deviation from the theoretical prediction. Various reasons have been suggested for human subjects' tendency to cooperate in the Centipede game; these include mistakes arising from bounded rationality, other-regarding preferences, direct and indirect reciprocity, use of the Take-the-Best heuristic, and best-reply matching $[10,13,14]$. Various modified versions of the basic Centipede have been investigated, and some of them have yielded significantly earlier defection, but the published evidence does not show which of the various modifications increase early defection. The experiment reported in this article is designed to disentangle the potential psychological factors and clarify our understanding of early defection in Centipede games.

Comparatively little research has focused on more competitive versions of the Centipede game (Figures 2 and 3). Parco, Rapoport, and Stein [15] first introduced a nine-node Centipede game with three players, high payoff differences between players, and zero payoffs at the final exit node. This game was subsequently used in a number of experiments [16-18]. In a study of a high-stakes version of the game, Rapoport, et al. [18], found very low levels of cooperation (almost $40 \%$ of their games ended at Node 1) and strong learning effects with decreasing cooperation across 60 repeated rounds of the game. This experiment elicited a different pattern of decisions from what is typically observed in standard Centipede games, but it remains unclear to which aspects of the design these differences can be attributed. The zero payoffs at the end are likely to have played an important role, but the number of players and the high payoff inequality may also have had large effects. Another confounding factor that could explain the strong learning effects reported in multi-player Zero-end Centipede games is the comparatively small pool of players taking part in the reported experiments, especially taking into account the high number of players per game and the numerous game repetitions (60-90 rounds). Even with anonymous random re-pairing after each round, players in these studies must have been aware of the high likelihood of repeated pairings with the same co-players, which could have elicited elements of supergame thinking and behavioral adaptations based on experience from previous rounds.

Three studies - two of which as yet unpublished - used Take-it-or-leave-it Centipede games with even greater payoff inequality than the games used by Rapoport and his colleagues $[4,19]$. In a complex 
research design investigating decision making in Take-it-or-leave-it Centipede games with partially unknown payoff functions, continuous versus discrete moves, and simultaneous versus sequential move order, Cox and James [19] reported the lowest cooperation levels ever reported in any type of Centipede game. In their version of the game with continuous and simultaneous moves, more than $95 \%$ of all games ended at the first decision node. However, as in the case of the multi-player Zero-end games discussed above, it is difficult to pinpoint which of their design aspects - continuous moves, partial payoff information, Zero-end payoffs, or Take-it-or-leave-it payoff function-were the decisive factors yielding these remarkable results. In a follow-up study [20], the same authors compared four-node Exponential Centipede games with two Take-it-or-leave-it game variations — one under time pressure and one without—and found that the high payoff difference of the Take-it-or-leave-it games decreased cooperation whereas time pressure did not affect the choices in the game. Nevertheless, due to the shortness of the games used in this experiment, overall stake sizes remained small (maximum of \$6.40) which is likely to have influenced decision making. Given the lack of conclusive results from previous studies on competitive Centipede games, a controlled comparison of a standard Centipede game with versions characterized by zero payoff ends and/or high player inequality is needed to disentangle the respective influences of these different game variations.

In order to resolve these ambiguities, the present study compared an eight-node version of McKelvey and Palfrey's [10] original Exponential Centipede game with three competitive variations: an Exponential Zero-end version, a Take-it-or-leave-it version, and a game combining both competitive features. Based on game-theoretic predictions, if the standard common knowledge assumption of game theory applies, then all games should elicit the same decision patterns, since they share the subgame perfect Nash equilibrium in which Player A defects at the first decision node. Nevertheless, the previous studies reviewed above indicate that certain game features may have important psychological effects on human decision makers, leading to strikingly different choices in games with only slightly different payoff functions. Drawing on this research, we propose the following two hypotheses:

Hypothesis 1. The standard Exponential Centipede game with positive payoffs at the terminal exit node will yield significantly higher levels of cooperation than Zero-end and Take-it-or-leave-it games.

Hypothesis 2. The game version combining both competitive features-Zero-end and Take-it-orleave-it payoff function - will yield even lower levels of cooperation than games with just one of these competitive features.

\section{Results and Discussion}

Out of 820 games played in total, 18 (2.2\%) ended at the first exit node and 21 (2.6\%) continued until the final exit node (Node 9). Figure 5 shows the proportions of games ending at each exit node per treatment condition. The modal exit node for Conditions 1 and 3 (both characterized by positive payoffs at the terminal node) was Node 6. The modal exit node for Conditions 2 and 4 (Zero-end games) was Node 5. Only $2 \%$ of all games in Condition 4 (Take-it-or-leave-it plus Zero-end game) and not a single game in Condition 2 (Exponential Zero-end game) continued beyond Node 7. Furthermore, Condition 2 yielded a very high percentage of games terminating in the first third of the game tree: Over $24 \%$ of 
games ended at one of the first three decision nodes, whereas in the other three conditions, this percentage did not exceed $4.5 \%$.

Exponential Centipede game

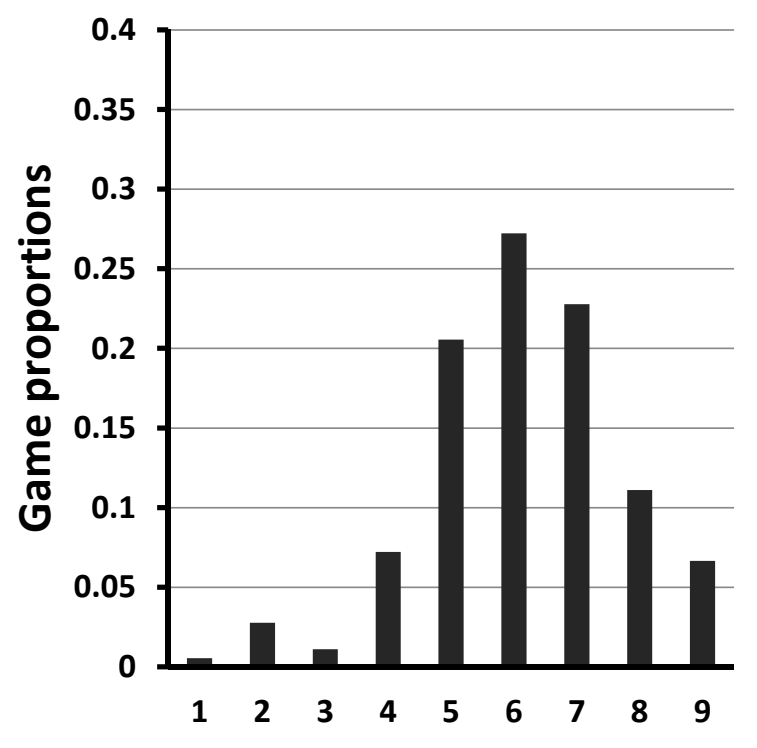

Exit nodes

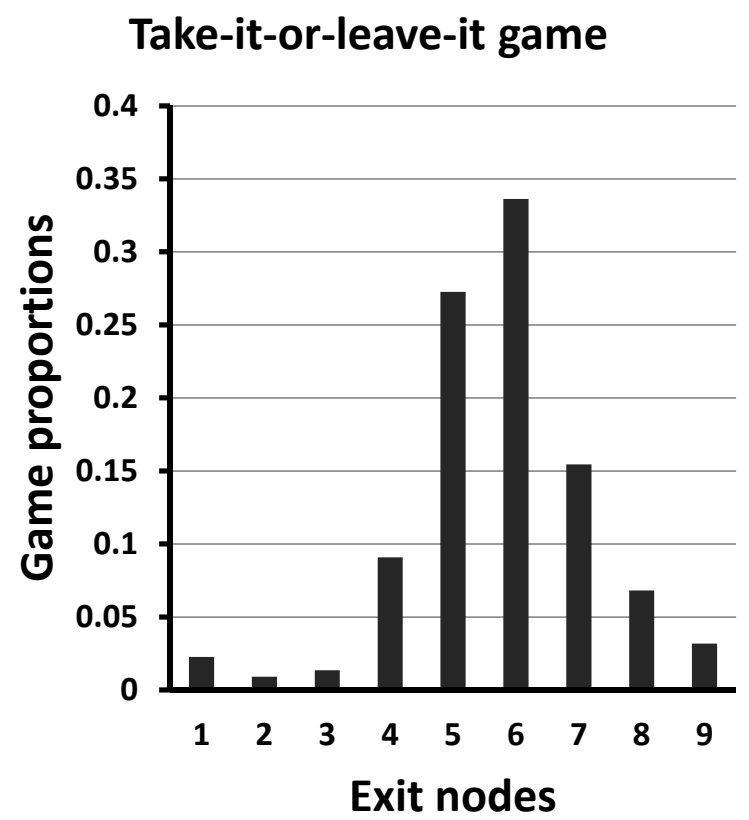

\section{Exponential Zero-end game}

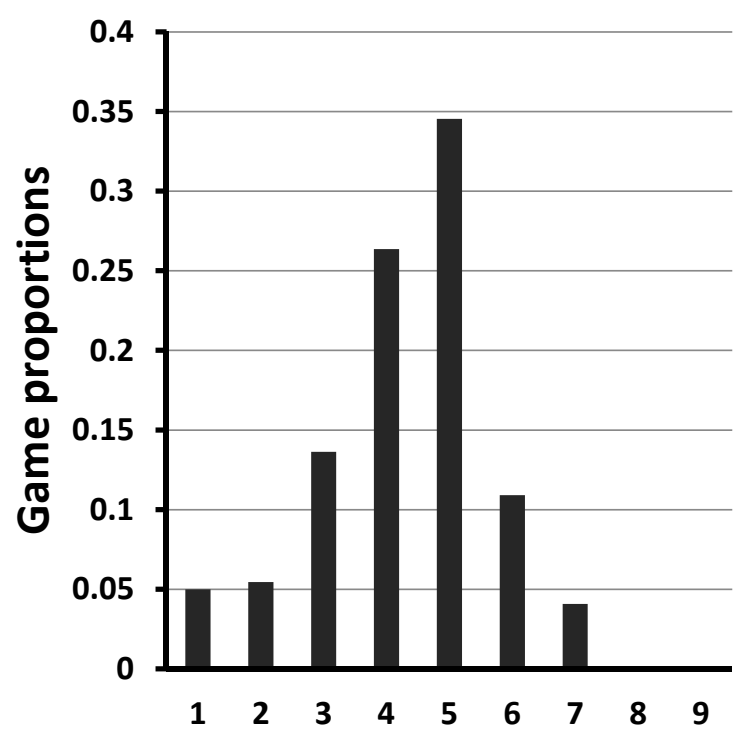

Exit nodes

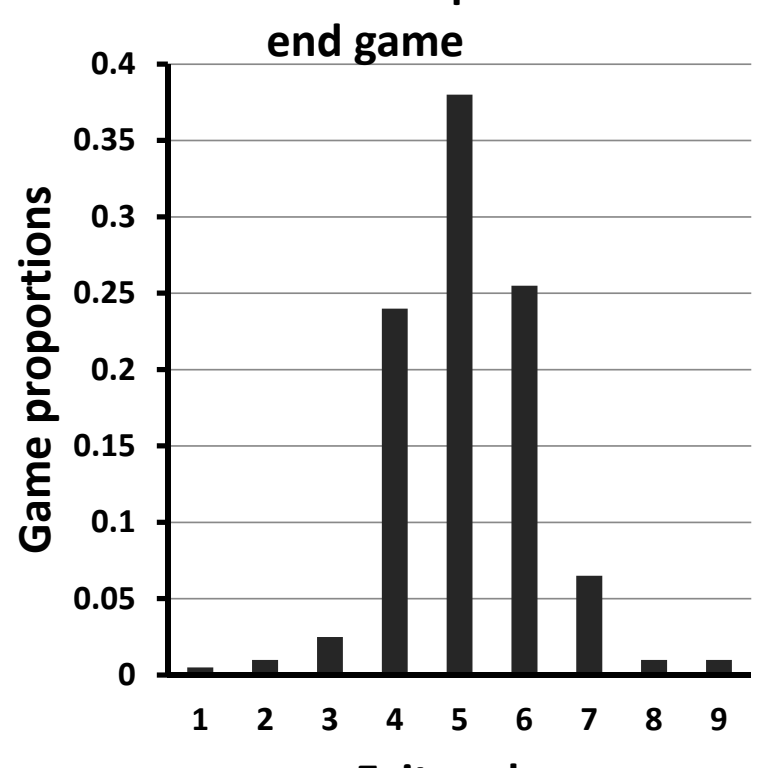

Exit nodes

Figure 5. Proportion of games ending at each exit node for the four game conditions.

Figure 6 displays the mean exit node per game round across the four treatment conditions. The learning curves for the different games do not show any discernible trends of either increasing or decreasing cooperation with greater experience in the game. 


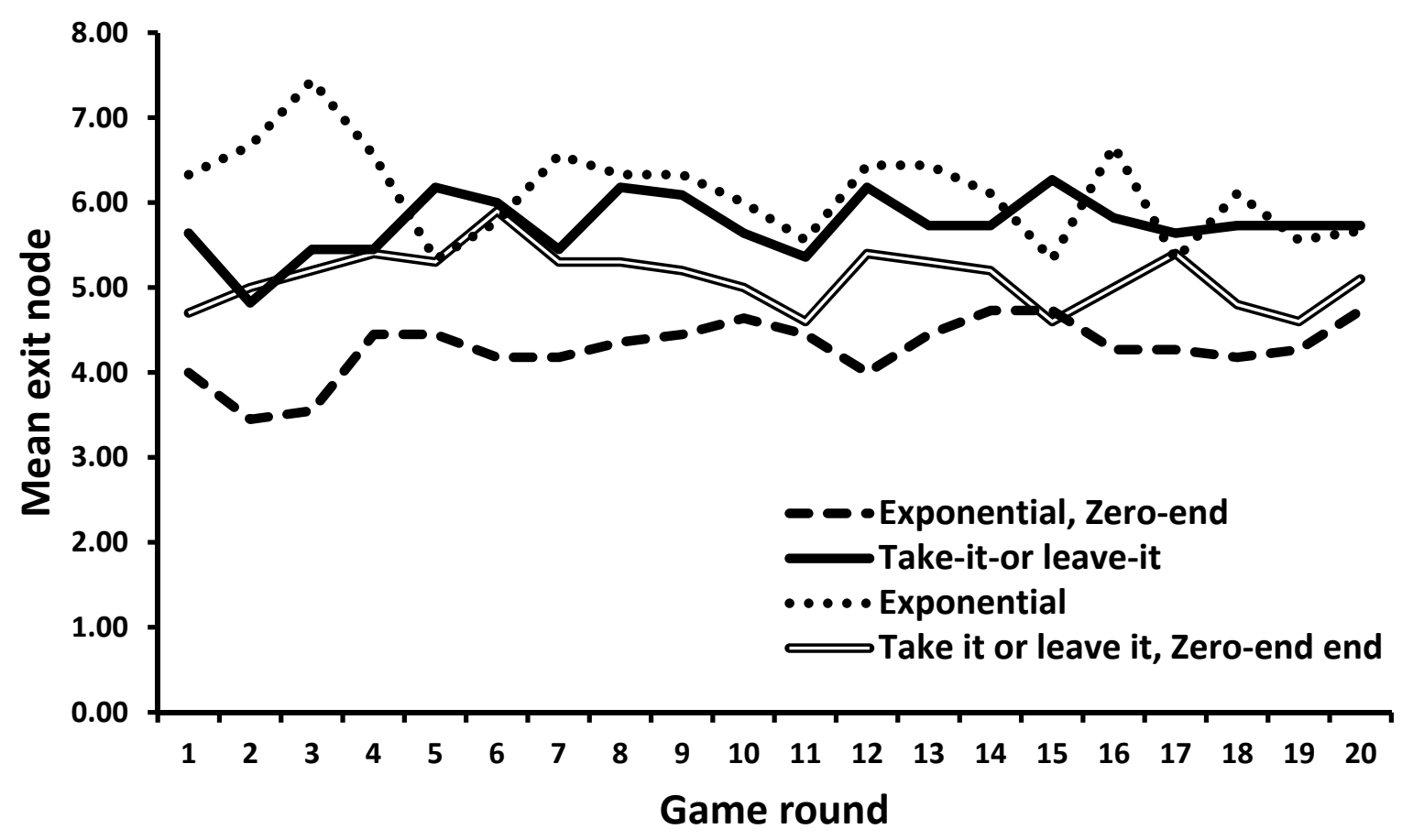

Figure 6. Mean exit nodes per game round across the four treatment conditions.

The experimental manipulation had a significant effect on the mean exit points, $F(3,76)=77.272$, $p<0.001$, eta squared $=0.75$ (medium effect size). Post-hoc comparison indicated significant differences between all four treatment conditions (Tukey HSD tests yielded $p<0.02$ in all cases). The Exponential game with positive payoffs at the end produced the highest cooperation levels $(M=6.13 ; S D=0.56)$. This was followed by the Take-it-or-leave-it Centipede game with positive payoffs at the end $(M=5.74$; $S D=0.35)$ and the Take-it-or-leave-it plus Zero-end game $(M=5.12 ; S D=0.33)$. The Exponential Zero-end Centipede game yielded the lowest levels of cooperation $(M=4.29 ; S D=0.35)$.

There were no significant gender differences. Across treatment conditions, the mean exit nodes were 5.19 (male) and 5.33 (female), $t(80)=0.467, p=0.64$ (two-tailed), and for each of the four treatment conditions separately, the mean difference was very small, with $p>0.25$.

\section{Discussion}

This study set out to investigate psychological factors influencing decision making in repeated reciprocal interactions modeled by the Centipede game. Overall, the results confirmed previous findings of low defection percentages at the first decision node and an absence of any marked learning effects across games rounds $[10,14]$. The results further support Hypothesis 1 that modifications of the game's payoff function elicit significant changes in the subjects' decision patterns, even though the game-theoretic solution is identical in all game variations. As predicted, Zero-end Centipede games and those characterized by a Take-it-or-leave-it payoff functions were shown to elicit significantly lower levels of cooperation compared to the standard Exponential Centipede game.

In the Zero-end game, this finding can be attributed to the deterrent psychological effect of a zero payoff at the terminal exit node. In this version, players are forced to defect at some point during the game in order to avoid a payoff of zero at the game's natural end. This finding is in line with previous 
research by Rapoport et al. [18] and Murphy et al. [16,17], who reported play in their three-player, Zero-end Centipede game to be much more competitive than in standard Centipede games with positive values at the natural end, but those previous studies did not allow the separate effects of game variations to be compared.

In the Take-it-or-Leave it game, lower cooperation levels can be explained by the extreme player inequality and the higher risk involved in choosing a cooperative GO move; if the co-player terminates the game at the immediately following decision node, then the payoff to the player who previously cooperated is zero. The findings on the Take-it-or-leave-it game link in with previous research that found very low cooperation levels using a game of a similar winner-takes-all payoff function $[19,20]$ although, once again, the research design made it impossible to pinpoint the factor(s) that caused the low levels of cooperation.

Contrary to the predictions of Hypothesis 2, the game design combining both competitive featuresTake-it-or-leave-it payoff structure and Zero-end - was not the one to yield the lowest cooperation levels overall. Whereas subjects in this condition exited significantly earlier than in the standard Exponential game and the Take-it-or-leave-it game with positive payoffs at the natural end, the game condition eliciting the earliest exit moves was the Exponential Centipede game with Zero-end payoff structure. When comparing the numbers of games terminating at each exit node across treatment conditions, the Exponential Zero-end game stood out as the condition with the most STOP moves in the first third of the game tree, leading to the lowest mean exit node of our four game variations.

We propose the following explanation for why the Exponential Zero-end game yielded lower levels of cooperation than the Take-it-or-leave-it plus Zero-end version. The payoff function of the Take-it-orleave-it plus Zero-end version is heavily dominated by zeroes (the losing player never receives anything), and one consequence of this is that the zero payoffs at the end has less salience than in the Exponential Zero-end version, where these are the only zeros. Consequently, the deterrent psychological effect of the zero payoffs at the end may be comparatively smaller than in the version characterized by the typical Exponential payoff function.

A second possibility, not necessarily negating the first, is that fairness-seeking or inequality-averse individuals may perceive the equal payoffs of zero to both players as a desirable goal in a Centipede game with Take-it-or-leave-it plus Zero-end payoff function, because the payoffs at all other exit nodes create extreme inequality between the two players - a lot more so than in the standard Exponential Centipede game. Indeed, two of our subjects with the role of Player B in Condition 4 opted for GO at the final decision node, thus choosing equal payoffs of zero at Exit node 9 over a personal payoff of 51.20 with the co-player receiving nothing at Exit node 8 . These decisions must either be errors arising from bounded rationality or manifestations of inequality aversion in the form of a strong drive to achieve a fair outcome for both individuals.

Fey, McKelvey, and Palfrey [21] and Kawagoe and Takizawa [22] studied choices in constant-sum Centipede games with high payoff differences, increasingly unequal from one decision node to the next, and they found more frequent defection at the first decision node than we found in our experiment. In game-theoretic terms, any two-player constant-sum game is strictly competitive, and an implication of this is that it is impossible for both players to benefit from cooperation, as there is no social gain from cooperation. In our non-constant-sum Centipede games, by contrast, the payoff pot increases and both 
players can hope to benefit from reciprocal cooperation, hence it is hardly surprising that we observed less defection at the first decision node.

\section{Experimental Section}

\subsection{Subjects}

The sample consisted of 82 undergraduate psychology students from the University of Leicester-17 males and 65 females - with a mean age of 19.78 years $(S D=3.29)$, all of whom received course credits for participation. Additionally, all subjects were entered into a lottery, and one person per testing session had his or her payoff from a randomly selected game completed during the experiment converted to a cash payment in pounds sterling. There is evidence for the validity of this incentive system [23,24]. The mean cash remuneration of the four subjects selected was $£ 5.60(\$ 8.21)$.

\subsection{Design}

The subjects were randomly assigned to one of four treatment conditions varying in their game designs: (a) Exponential Centipede game; (b) Exponential Zero-end Centipede game; (c) Take-it-or-leave-it Centipede game; and (d) Take-it-or-leave-it plus Zero-end Centipede game (see Figures 1-4). The dependent variable was the mean exit node of all games completed during one round of the game. Large values indicated late exit moves and thus high levels of cooperation, whereas small values indicated early exit moves and low levels of cooperation.

\subsection{Materials}

The study was conducted in a large computer laboratory. Each subject was seated at a computerseparated by at least one free seat from the next person - and interacted in the Centipede game through a custom-made, web-based game application that included several detailed instruction slides and a color-coded, animated display of the relevant Centipede game.

\subsection{Procedure}

Testing took place in groups of 18 to 22 subjects with each session lasting between 30 and 45 min. The subjects were told not to communicate with each other, and the experimenter ensured that subjects focused attention on their own computer screens. After filling in the consent form, subjects were presented with the game instructions and encouraged to ask questions about the rules and procedure. They were then randomly assigned to a player role (Player A or B), and they remained in the same role for the duration of the testing session. Each subject completed 20 rounds of one of the four Centipede games, with random and anonymous pairings for each round. They received real-time feedback on their co-players' moves, the outcome of each game, and the number of rounds completed. Following the last round, one subject was randomly drawn from the group for remuneration on a randomly selected game played during the session. 


\section{Conclusions}

Taken together, the findings of this study shed further light on the different and often subtle aspects of reciprocal interactions that may influence the extent of cooperation and teamwork exhibited by human decision makers. The increase of inequality between two individuals in an interpersonal relationship was shown to reduce overall cooperation significantly. Furthermore, the introduction of undesirable final payoffs as a consequence of unconditional or automatic cooperation was shown to have an even stronger effect on cooperation. Surprisingly, however, coupling these two deterrent factors did not reinforce their respective effects but appeared to form a novel decision-making context that elicited slightly higher cooperation levels than expected. The results of our study therefore demonstrate the need for carefully controlled experimental designs when investigating decision making and cooperation. Variations in the game's payoff structure can elicit significant behavioral changes, and the interplay of these factors can have unexpectedly complex effects.

\section{Acknowledgments}

The research reported in this article was supported by an award from Friedrich-Naumann-Stiftung für die Freiheit. The authors are grateful to Kevin McCracken for help with software development.

\section{Author Contributions}

E.M.K. conceived and designed the experiment in consultation with B.D.P. and A.M.C., E.M.K performed the data-collection, E.M.K. in consultation with B.D.P. analyzed the data, and all authors contributed to the write-up.

\section{Conflicts of Interest}

The authors declare no conflict of interest.

\section{References}

1. Rosenthal, R.W. Games of perfect information, predatory pricing and chain store paradox. J. Econ. Theory 1981, 25, 92-100.

2. Binmore, K. Modeling rational players: Part 1. Econ. Philos. 1987, 3, 179-214.

3. Reny, P.J. Rationality in extensive-form games. J. Econ. Perspect. 1992, 6, 103-118.

4. Huck, S.; Jehiel, P. Public Statistics and Private Experience: Varying Feedback Information in a Take-or-Pass Game. Available online: http://www.enpc.fr/ceras/jehiel/huck_jehiel.pdf (accessed on 15 October 2013).

5. Aumann, R.J. Backward induction and common knowledge of rationality. Games Econ. Behav. 1995, 8, 6-19.

6. Aumann, R.J. On the Centipede game. Games Econ. Behav. 1998, 23, 97-105.

7. Ben-Porath, E. Rationality, Nash and backwards equilibrium induction in perfect-information. Rev. Econ. Stud. 1997, 64, 23-46. 
8. Zauner, K.G. A payoff uncertainty explanation of results in experimental centipede games. Games Econ. Behav. 1999, 26, 157-185.

9. Bornstein, G.; Kugler, T.; Ziegelmeyer, A. Individual and group decisions in the Centipede game: Are groups more "rational" players? J. Exp. Soc. Psychol. 2004, 40, 599-605.

10. McKelvey, R.D.; Palfrey, T.R. An experimental study of the Centipede game. Econometrica 1992, 60, 803-836.

11. Nagel, R.; Tang, F.F. Experimental results on the Centipede game in normal form: An investigation on learning. J. Math. Psychol. 1998, 42, 356-384.

12. Pulford, B.D.; Krockow, E.M.; Colman, A.M.; Lawrence, C.L. University of Leicester: Leicester, UK. Unpublished work, 2015.

13. Droste, E.; Kosfeld, M.; Voorneveld, M. Best-reply matching in games. Math. Soc. Sci. 2003, 46, 291-309.

14. Pulford, B.D.; Colman, A.M.; Lawrence, C.L.; Krockow, E.M. University of Leicester: Leicester, UK. Unpublished work, 2015.

15. Parco, J.E.; Rapoport, A.; Stein, W.E. Effects of financial incentives on the breakdown of mutual trust. Psychol. Sci. 2002, 13, 292-297.

16. Murphy, R.O.; Rapoport, A.; Parco, J.E. Population learning of cooperative behavior in a three-person centipede game. Ration. Soc. 2004, 16, 91-120.

17. Murphy, R.O.; Rapoport, A.; Parco, J.E. The breakdown of cooperation in iterative real-time trust dilemmas. Exp. Econ. 2006, 9, 147-166.

18. Rapoport, A.; Stein, W.E.; Parco, J.E.; Nicholas, T.E. Equilibrium play and adaptive learning in a three-person Centipede game. Games Econ. Behav. 2003, 43, 239-265.

19. Cox, J.C.; James, D. Clocks and trees: Isomorphic Dutch auctions and centipede games. Econometrica 2012, 80, 883-903.

20. Cox, J.C.; James, D. On replication and perturbation of the McKelvey and Palfrey Centipede game experiment. In Research in Experimental Economics, Volume 18: Replication in Economic Experiments; Deck, C.A., Fatas, E., Rosenblat, T., Eds.; Emerald: Bingley, UK, 2015.

21. Fey, M.; McKelvey, R.D.; Palfrey, T.R. An experimental study of constant-sum centipede games. Int. J. Game Theory 1996, 25, 269-287.

22. Kawagoe, T.; Takizawa, H. Level- $k$ analysis of experimental centipede games. J. Econ. Behav. Organ. 2012, 82, 548-566.

23. Cubitt, R.; Starmer, C.; Sugden, R. On the validity of the random lottery incentive system. Exper. Econ. 1998, 1, 115-131.

24. Bolle, F. High reward experiments without high expenditure for the experimenter. J. Econ. Psychol. 1990, 11, 157-167.

(C) 2015 by the authors; licensee MDPI, Basel, Switzerland. This article is an open access article distributed under the terms and conditions of the Creative Commons Attribution license (http://creativecommons.org/licenses/by/4.0/). 